\title{
Análise da Recorrência de Fibrilação Atrial durante Terapia com Sotalol ou Quinidina
}

\author{
Henrique Horta Veloso, Angelo Amato Vincenzo de Paola, pelos Investigadores da SOCESP \\ São Paulo, SP
}

\begin{abstract}
Objetivo - Analisar a incidência e as características das crises de recorrência de fibrilação atrial (FA) em pacientes sob terapia com sotalol ou quinidina.

Métodos - Cento e vinte e um pacientes receberam de forma randômica sotalol (58 pacientes) ou quinidina (63 pacientes) após reversão de FA paroxística e foram acompanhados, ambulatorialmente, por 6 meses. As sintomatologias e as freqüências ventriculares ao eletrocardiograma de 12 derivações da crise inicial e da recorrência foram comparadas entre os grupos de drogas. As características clínicas e os dados do ecocardiograma foram analisados como preditores de recorrência.

Resultados - Dezessete (14\%) pacientes apresentaram recorrência da arritmia, 7 (12\%) em uso de sotalol e 10 (16\%) em uso de quinidina. A recorrência ocorreu mais tardiamente no grupo tratado com sotalol (mediana de 69 dias) em comparação ao grupo tratado com quinidina (mediana de 10 dias) $(p=0,04)$. A sintomatologia esteve presente em 14 (82\%) pacientes na crise inicial e em $8(47 \%)$ pacientes na recorrência. O tratamento antiarritmico proporcionou recorrências menos sintomáticas ( $p<0,04)$, não sendo possível demonstrar diferença significante entre as terapias. Somente os pacientes tratados com sotalol apresentaram menor freqüência ventricular na recorrência em comparação à crise inicial $(p<0,02)$. Todas as variáveis falharam em predizer recorrência da FA.

Conclusão - Não foi possível demonstrar diferença entre o sotalol e a quinidina na prevenção de FA. O tratamento antiarrítmico proporcionou a ocorrência de recorrências menos sintomáticas. Os pacientes tratados com sotalol apresentaram recorrência mais tardiamente e freqüencia ventricular significantemente menor na recorrência em comparação à crise inicial.
\end{abstract}

Palavras-chave: fibrilação atrial, recorrência, sotalol, quinidina

\section{Analysis of Atrial Fibrillation Recurrence during Therapy with Sotalol or Quinidine}

Purpose - To analyze the recurrences of atrial fibrillation in patients treated with sotalol or quinidine.

Methods - After conversion to sinus rhythm, $121 \mathrm{pa}$ tients with paroxysmal atrial fibrillation were randomized to sotalol (58 patients) or quinidine (63 patients) and followed-up during 6 months. Symptoms and ventricular rates on the 12 lead electrocardiogram of the arrhythmic events were compared between the two groups. Clinical and echocardiographic characteristics were analyzed as predictors of atrial fibrillation recurrence.

Results - Seventeen (14\%) patients relapsed into atrial fibrillation; 7 (12\%) were treated with sotalol and $10(16 \%)$ with quinidine. Recurrence occurred later in the sotalol group (median 69 days) in comparison with the quinidine group (median 10 days) ( $p=0.04$ ). Symptoms were present in 14 (82\%) patients during the initial crisis and in 10 (47\%) during recurrence. Recurrence was less symptomatic during antiarrhythmic therapy $(p<0.04)$, with no statistical differences between the two groups. Only patients treated with sotalol had ventricular rates during the recurrences lower than during initial crisis $(p<0.02)$. All variables failed to predict recurrence of atrial fibrillation.

Conclusions - It was not possible demonstrate differences between sotalol and quinidine for the prevention of atrial fibrillation. Recurrence was less symptomatic during antiarrhythmic therapy. Patients treated with sotalol relapsed to atrial fibrillation later and had ventricular rates during recurrences significantly lower than during the initial crisis.

Key-words: atrial fibrillation, recurrence, sotalol, quinidine

Arq Bras Cardiol, volume 70 (n 1), 43-49, 1998

Escola Paulista de Medicina - UNIFESP

Correspondência: Henrique Horta Veloso - Setor de Eletrofisiologia Clínica EPM - UNIFESP - Rua Napoleão de Barros, 593 - 04024-002 - São Paulo, SP Recebido para publicação em 1/8/97

Aceito em 23/10/97
A fibrilação atrial (FA) é a arritmia sustentada mais comum. Devido à morbidade e à mortalidade às quais se associa ${ }^{1}$, deve, sempre que possível, ser revertida para o ritmo sinusal. Nos casos com insucesso na restauração do ritmo 
sinusal, a freqüência ventricular deve ser controlada, através da administração de drogas como o digital, antagonistas dos canais de cálcio ou betabloqueadores.

A quinidina, droga da classe IA de Vaughan-Williams, com ação bloqueadora dos canais de sódio, é um dos fármacos utilizados na terapêutica da FA, tanto na reversão das crises da arritmia, quanto na prevenção de recorrências. Devido à sua ação vagolítica, pode levar a um aumento da freqüência ventricular durante a FA, necessitando muitas vezes do uso concomitante de outros fármacos bloqueadores da condução atrioventricular.

O d,1-sotalol é uma droga betabloqueadora que apresenta também a capacidade de aumentar a duração do potencial de ação da célula cardíaca, bloqueando os canais de potássio, portanto, com propriedades das classes II e III de Vaughan-Williams ${ }^{2}$, sendo utilizado principalmente no tratamento de arritmias ventriculares. A sua utilização no tratamento da FA foi, inicialmente, indicada para a conversão da arritmia no pós-operatório precoce da cirurgia de revascularização miocárdica, quando se mostrou superior ao digital e à disopiramida ${ }^{3}$.

O sotalol mostrou-se tão eficaz quanto a quinidina na manutenção do ritmo sinusal, após cardioversão elétrica, em pacientes com FA crônica ${ }^{4}$. Neste estudo, o sotalol foi bem tolerado e controlou a freqüência ventricular dos pacientes que apresentaram recorrência.

Diversas outras drogas antiarrítmicas como a disopiramida, procainamida, propafenona, verapamil e amiodarona têm sido utilizadas na prevenção de recorrências. Entretanto, até o momento, não existe consenso quanto à medicação de escolha em portadores de FA paroxística ${ }^{5-8}$. Por este motivo, considera-se como melhor opção terapêutica o medicamento melhor tolerado, com menos efeitos colaterais, e que, caso o paciente apresente recorrência da arritmia, esta se faça de forma menos sintomática e com freqüência ventricular controlada. A amiodarona parece ser a melhor opção nos casos refratários às outras drogas e nos pacientes com átrios aumentados ${ }^{9}$.

O objetivo deste estudo foi comparar duas drogas, sotalol e quinidina, quanto à incidência de recorrências de FA, após reversão de crise paroxística. Também foram analisados o tempo até a crise recorrente de FA, o comportamento da freqüência ventricular e a sintomatologia dos pacientes com recorrência da arritmia.

\section{Métodos}

Cento e vinte e um pacientes, com idades de 25 a 75 anos, após reversão de episódio paroxístico de FA, foram selecionados em 18 centros cardiológicos no Brasil, sendo acompanhados por seis meses. A FA poderia estar ou não associada a cardiopatias estruturais. Os critérios de inclusão e exclusão estão expostos nas tabelas I e II. As características clínicas dos pacientes encontram-se na tabela III e os dados do ecocardiograma na tabela IV.

Os pacientes incluídos no estudo foram randomizados para receberem uma das opções de tratamento: 1) sulfato de
Tabela I - Critérios de inclusão

1) Paciente sintomático com episódio paroxístico de fibrilação atrial revertido em período não $>48 \mathrm{~h}$ antes de sua inclusão no estudo.

2) Idade entre 20 e 75 anos.

3) Mulheres com potencial para engravidar deveriam apresentar testes de gravidez negativos 2 dias antes do início do tratamento, repetidos 2 vezes ao acompanhamento.

\begin{tabular}{|l|}
\hline \multicolumn{1}{|c|}{ Tabela II - Critérios de exclusão } \\
\hline 1) Insuficiência cardíaca moderada ou severa (classes III ou IV da New York \\
Heart Association) \\
2) Alergia, hipersensibilidade ou contra-indicação às drogas do estudo. \\
3) Freqüência cardíaca média $<50 \mathrm{bpm}$. \\
4) Pressão arterial sistólica $<100 \mathrm{mmHg}$. \\
5) Pressão arterial diastólica $>110 \mathrm{mmHg}$. \\
6) Uso abusivo de drogas ou álcool. \\
7) Gravidez, lactação ou não uso de métodos contraceptivos adequados. \\
8) Insuficiência renal com clearance de creatinina $>30 \mathrm{ml} /$ min ou potássio \\
$<3,8$ nmol/l. O clearance de creatinina deveria ser realizado se a creatinina \\
sérica fosse $>2,5 \mathrm{mg} /$ dl. \\
9) Fração de ejeção, pelo método de Pombo ao ecocardiograma, $\leq 40 \%$. \\
10) Diâmetro de átrio esquerdo $>5,2 \mathrm{~cm}$ ao ecocardiograma. \\
11) Paciente sob terapia com drogas antiarrítmicas ou betabloqueadores \\
12) Uso de amiodarona nos últimos 45 dias. \\
13) Infarto do miocárdio confirmado por eletrocardiograma ou prova enzimática \\
no último mês. \\
14) Uso de quinidina ou sotalol no último mês. \\
15) Qualquer doença, condição ou terapia que pudesse trazer risco ao paciente \\
ou interferisse no resultado do estudo.
\end{tabular}

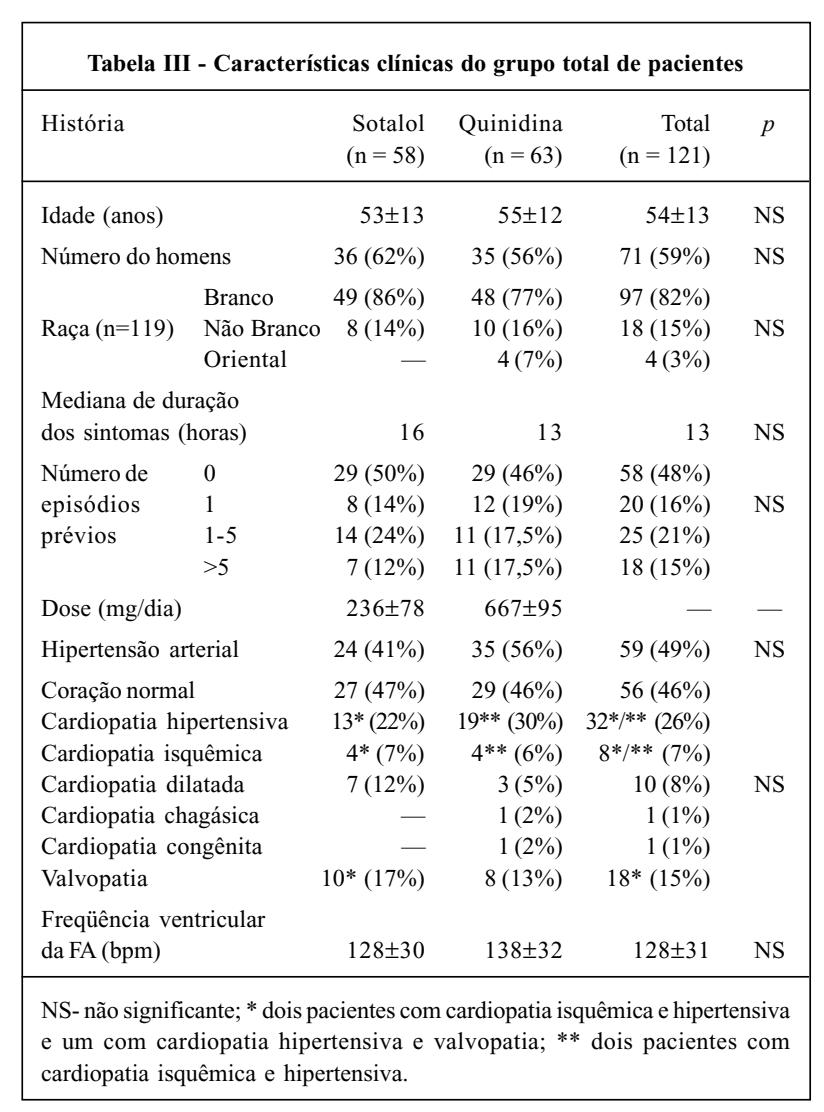




\begin{tabular}{|lcccc|}
\hline \multicolumn{5}{|c|}{ Tabela IV - Dados do ecocardiograma do grupo total de pacientes } \\
\hline & Sotalol & Quinidina & Total & $p$ \\
\hline Átrio esquerdo (mm) & $38 \pm 6$ & $40 \pm 5$ & $39 \pm 6$ & NS \\
DDVE (mm) & $50 \pm 7$ & $50 \pm 8$ & $50 \pm 7$ & NS \\
DSVE (mm) & $33 \pm 7$ & $31 \pm 6$ & $32 \pm 6$ & NS \\
Fração de ejeção (\%) & $66 \pm 10$ & $69 \pm 9$ & $68 \pm 9$ & NS \\
\hline Dados em média \pm desvio padrão; DDVE- diâmetro diastólico final de \\
ventrículo esquerdo; DSVE- diâmetro sistólico final de ventrículo esquerdo. \\
\hline \multicolumn{5}{l}{} \\
\hline
\end{tabular}

\begin{tabular}{|c|c|c|c|c|}
\hline & Sotalol & Quinidina & Total & $p$ \\
\hline Número de pacientes & 7 & 10 & 17 & NS \\
\hline $\begin{array}{l}\text { Mediana da recorrência (dias) } \\
\text { Freqüência ventricular da }\end{array}$ & 69 & 10 & 14 & 0,04 \\
\hline crise inicial (bpm) & $98 \pm 18$ & $102 \pm 28$ & $100 \pm 23$ & NS \\
\hline $\begin{array}{l}\text { Freqüência ventricular da } \\
\text { recorrência (bpm) }\end{array}$ & $82 \pm 20$ & $113 \pm 44$ & $99 \pm 38$ & NS \\
\hline $\mathrm{p}$ & 0,018 & 0,059 & NS & \\
\hline
\end{tabular}

quinidina $200 \mathrm{mg}$ quatro vezes ao dia, podendo ser modificado para 200mg três vezes ao dia, ou 2) d,1-sotalol 160mg duas vezes ao dia, podendo ser modificado para $80 \mathrm{mg}$ duas ou três vezes ao dia (fig. 1). Drogas betabloqueadoras ou outros antiarrítmicos não foram permitidos como medicação concomitante.

Os pacientes foram acompanhados ao nível ambulatorial com consultas uma semana após à reversão e, mensalmente, até o $6^{\circ}$ mês. Eletrocardiogramas (ECG) de 12 derivações foram realizados por ocasião da admissão hospitalar e durante as consultas durante o acompanhamento. Um ecocardiograma foi realizado no início do estudo, com medidas do tamanho do átrio esquerdo (AE), diâmetros diastólico e sistólico finais e fração de ejeção de ventrículo esquerdo (VE). Os pacientes foram orientados a retornarem

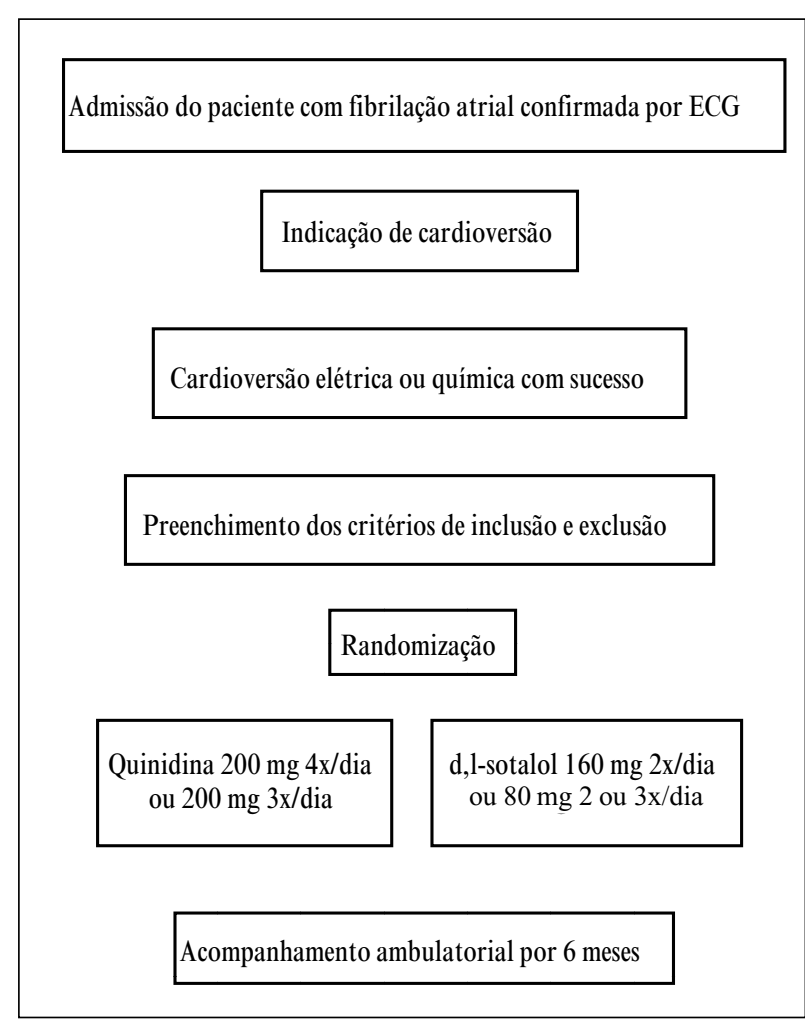

Fig. 1 - Fluxograma de inclusão de pacientes

para consulta caso manifestassem sintomatologia compatível com recorrência da arritmia ou efeitos possivelmente relacionados à terapia administrada. Eventuais recorrências que pudessem ocorrer de maneira assintomática seriam flagradas nas consultas de rotina. Em todas consultas, além de um exame clínico minucioso, um questionário objetivo era preenchido quanto à sintomatologia apresentada.

A sintomatologia e as freqüências ventriculares calculadas ao ECG na crise inicial e na recorrência foram comparadas entre os grupos sotalol e quinidina. As características clínicas e dados do ecocardiograma

\begin{tabular}{|c|c|c|c|c|c|c|}
\hline \multicolumn{7}{|c|}{ Tabela VI - Características dos pacientes com recorrência } \\
\hline Paciente & Sexo & Idade (anos) & Cardiopatia & Droga & Dose (mg/dia) & Dia da recorrência \\
\hline 1 & Masculino & 62 & Hipertensiva & Quinidina & 800 & 12 \\
\hline 2 & Masculino & 39 & Dilatada & Sotalol & 160 & 33 \\
\hline 3 & Feminino & 69 & Coração normal & Quinidina & 800 & 7 \\
\hline 4 & Feminino & 61 & Hipertensiva & Quinidina & 800 & 8 \\
\hline 5 & Feminino & 58 & Coração normal & Quinidina & 800 & 8 \\
\hline 6 & Feminino & 70 & Valvopatia mitral & Quinidina & 600 & 24 \\
\hline 7 & Feminino & 67 & Hipertensiva & Quinidina & 600 & 126 \\
\hline 8 & Masculino & 50 & Dilatada & Sotalol & 240 & 7 \\
\hline 9 & Feminino & 65 & Coração normal & Sotalol & 320 & 106 \\
\hline 10 & Feminino & 67 & Hipertensiva & Quinidina & 800 & 14 \\
\hline 11 & Feminino & 73 & Coração normal & Sotalol & 320 & 102 \\
\hline 12 & Masculino & 73 & Prolapso mitral & Sotalol & 160 & 47 \\
\hline 13 & Feminino & 57 & Hipertensiva & Quinidina & 800 & 7 \\
\hline 14 & Masculino & 42 & Valvopatia aórtica & Sotalol & 240 & 69 \\
\hline 15 & Feminino & 67 & Valvopatia mitral & Sotalol & 320 & 125 \\
\hline 16 & Masculino & 44 & Dilatada & Quinidina & 800 & 12 \\
\hline 17 & Feminino & 68 & Coração normal & Quinidina & 800 & 4 \\
\hline
\end{tabular}




\begin{tabular}{|c|c|c|c|}
\hline \multicolumn{4}{|c|}{ Tabela VII - Sintomatologia das crises de fibrilação atrial } \\
\hline Paciente & Sintomas da crise inicial & Sintomas da recorrência & Resultado \\
\hline 1 & síncope & - & melhorado \\
\hline 2 & palpitação & - & melhorado \\
\hline 3 & palpitação, dispnéia & tontura, fraqueza & inalterado \\
\hline 4 & vertigem, palpitação & - & melhorado \\
\hline 5 & vertigem, palpitação & fraqueza & melhorado \\
\hline 6 & - & - & inalterado \\
\hline 7 & - & - & inalterado \\
\hline 8 & fadiga, palpitação & fraqueza & melhorado \\
\hline 9 & palpitação, vertigem, precordialgia & - & melhorado \\
\hline 10 & palpitação, dispnéia & taquicardia, dispnéia & inalterado \\
\hline 11 & palpitação & palpitação & inalterado \\
\hline 12 & vertigem, dispnéia, precordialgia & palpitação, dispnéia & melhorado \\
\hline 13 & - & - & inalterado \\
\hline 14 & vertigem, fadiga & - & melhorado \\
\hline 15 & síncope, vertigem, palpitação, dispnéia, precordialgia & tontura, palpitação & melhorado \\
\hline 16 & dispnéia & - & melhorado \\
\hline 17 & vertigem & tontura & inalterado \\
\hline
\end{tabular}

\begin{tabular}{|c|c|c|c|c|}
\hline \multicolumn{5}{|c|}{$\begin{array}{c}\text { Tabela VIII - Comparação da sintomatologia entre a crise inicial e a } \\
\text { recorrência }\end{array}$} \\
\hline \multirow[t]{2}{*}{ Droga } & \multicolumn{2}{|c|}{ Sintomatologia entre as crises } & \multirow[t]{2}{*}{ Total } & \multirow[t]{2}{*}{$\mathrm{p}$} \\
\hline & Melhorado & Inalterado & & \\
\hline Sotalol & $6(86 \%)$ & $1(14 \%)$ & $7(100 \%)$ & 0,08 \\
\hline Quinidina & $4(40 \%)$ & $6(60 \%)$ & $10(100 \%)$ & \\
\hline Total & $10(59 \%)$ & $7(41 \%)$ & $17(100 \%)$ & \\
\hline
\end{tabular}

foram testados como preditores de recorrência de FA.

Todos os pacientes assinaram termo de consentimento informado. Foram seguidas as recomendações da Declaração de Helsinki para pesquisas biomédicas envolvendo seres humanos e o estudo foi aprovado pelas comissões de ética médica dos respectivos hospitais.

Para análise estatística foram utilizados os testes $t$ de Student (Gosset), quiquadrado, Mann-Whitney, Wilcoxon e Kruskall-Wallis. Foram considerados significantes valores dep $<0,05$.

\begin{tabular}{|c|c|c|c|c|c|}
\hline \multirow[t]{2}{*}{ História } & & \multicolumn{2}{|c|}{ Recorrência } & \multirow[t]{2}{*}{ Total } & \multirow[t]{2}{*}{$\mathrm{p}$} \\
\hline & & $\operatorname{Sim}$ & Não & & \\
\hline Número de pacientes & & $17(14 \%)$ & $104(86 \%)$ & $121(100 \%)$ & - \\
\hline Idade (anos) & & $61 \pm 11$ & $53 \pm 12$ & $54 \pm 13$ & NS \\
\hline \multirow[t]{3}{*}{ Sexo } & Masculino & $6(35 \%)$ & $65(62,5 \%)$ & $71(59 \%)$ & 0,06 \\
\hline & Feminino & $11(65 \%)$ & $39(37,5 \%)$ & $50(41 \%)$ & \\
\hline & Branco & $14(82 \%)$ & $83(81 \%)$ & $97(82 \%)$ & \\
\hline Raça & Não Branco & $2(12 \%)$ & $16(16 \%)$ & $18(15 \%)$ & NS \\
\hline$(\mathrm{n}=119)$ & Oriental & $1(6 \%)$ & $3(3 \%)$ & $4(3 \%)$ & \\
\hline \multicolumn{6}{|l|}{ Mediana de duração dos sintomas } \\
\hline de fibrilação atrial (horas) & & 87 & 13 & 13 & NS \\
\hline Número de & 0 & $6(35 \%)$ & $52(50 \%)$ & $58(48 \%)$ & \\
\hline episódios & 1 & $5(29 \%)$ & $15(14,5 \%)$ & $20(16 \%)$ & NS \\
\hline \multirow[t]{2}{*}{ prévios } & $1-5$ & $3(18 \%)$ & $22(21 \%)$ & $25(21 \%)$ & \\
\hline & $>5$ & $3(18 \%)$ & $15(14,5 \%)$ & $18(15 \%)$ & \\
\hline Hipertensão arterial & & $9(53 \%)$ & $50(48 \%)$ & $59(49 \%)$ & NS \\
\hline Coração normal & & $5(29 \%)$ & $51(49 \%)$ & $56(46 \%)$ & \\
\hline Cardiopatia hipertensiva & & $5(29 \%)$ & $27 *(26 \%)$ & $32 *(26 \%)$ & \\
\hline Cardiopatia isquêmica & & - & $8 *(8 \%)$ & $8 *(7 \%)$ & \\
\hline Cardiopatia dilatada & & $3(18 \%)$ & $7(7 \%)$ & $10(8 \%)$ & NS \\
\hline Cardiopatia chagásica & & - & $1(1 \%)$ & $1(1 \%)$ & \\
\hline Cardiopatia congênita & & - & $1(1 \%)$ & $1(1 \%)$ & \\
\hline Valvopatia & & $4(24 \%)$ & $14 *(13 \%)$ & $18 *(15 \%)$ & \\
\hline Freqüência ventricular da FA (bpm) & & $112 \pm 31$ & $131 \pm 31$ & $128 \pm 31$ & NS \\
\hline
\end{tabular}




\begin{tabular}{|lcccc|}
\hline \multicolumn{5}{|c|}{ Tabela X - Dados do ecocardiograma dos pacientes com recorrência } \\
de fibrilação atrial
\end{tabular}

Dados em média \pm desvio padrão; DDVE- diâmetro diastólico final de ventrículo esquerdo; DSVE- diâmetro sistólico final de ventrículo esquerdo.

\section{Resultados}

Dos 121 pacientes do estudo, 17 apresentaram recorrência da arritmia nos seis meses de acompanhamento, correspondendo a $14 \%$ do total. Ocorreram $7(12 \%)$ casos de recorrência nos 58 pacientes tratados com sotalol, e 10 (16\%) nos 63 pacientes tratados com quinidina. Não houve diferença significante entre os grupos tratados com sotalol e quinidina quanto à incidência de recorrência (tab. V). As características dos pacientes com recorrência estão listadas na tabela VI.

Os pacientes apresentaram recorrência da FA de quatro a 126 dias após o início do tratamento, mediana de 14 dias. A recorrência ocorreu entre os dias sete e 125 nos tratados com sotalol, mediana de 69 dias; e entre os dias quatro e 126 nos tratados com quinidina, mediana de 10 dias. Os pacientes tratados com sotalol apresentaram recorrência significantemente mais tardia $(p=0,04)$ (tab. V).

Não houve diferença significante entre os diversos grupos de cardiopatia quanto ao tempo de recorrência da $\operatorname{arritmia}(p=0,10)$. A mediana de recorrência dos cinco pacientes com FA isolada foi de oito dias, dos cinco portadores de cardiopatia hipertensiva de 14 dias, dos quatro valvopatas de 58 dias e dos três portadores de cardiomiopatia dilatada de 12 dias.

Na crise inicial de FA, 14 (82\%) dos 17 pacientes que cursaram com recorrência apresentaram sintomas que puderam ser atribuídos à arritmia. A recorrência foi sintomática em $8(47 \%)$ destes pacientes. Independentemente do tipo da medicação antiarrítmica em uso, houve diminuição significante da sintomatologia por ocasião da recorrência $(\mathrm{p}=0,035)$. Os sintomas das crises de FA encontram-se listados na tabela VII.

Foi comparada a sintomatologia entre a crise inicial e a recorrência e os pacientes foram classificados como: melhorados - quando houve diminuição ou desaparecimento dos sintomas; inalterados - quando as crises cursaram com sintomatologia semelhante; e piorados - quando a recorrência foi mais sintomática. Houve melhora da sintomatologia em 10/17 (59\%) dos pacientes com recorrência, sendo 6/7 (86\%) dos tratados com sotalol e 4/10 (40\%) dos tratados com quinidina. Houve tendência da recorrência ser melhor tolerada nos pacientes tratados com sotalol
Recorrência de FA durante terapia com sotalol ou quinidina

$(\mathrm{p}=0,08)$. Em nenhum paciente ocorreu piora da sintomatologia (tab. VII e VIII).

As freqüências ventriculares foram de $100 \pm 24 \mathrm{bpm}$ na crise inicial e de $99 \pm 38 \mathrm{bpm}$ na recorrência no grupo total de pacientes, não havendo diferença significante $(p=0,65)$.

Os pacientes sob uso do sotalol apresentaram freqüência ventricular de $98 \pm 18 \mathrm{bpm}$ na crise inicial, havendo diminuição significante da freqüência ventricular na recorrência para $82 \pm 20 \mathrm{bpm}(\mathrm{p}=0,018)$. Os pacientes que receberam quinidina apresentaram freqüência ventricular de $102 \pm 28$ bpm na crise inicial e $112 \pm 44 \mathrm{bpm}$ na recorrência, com clara tendência a um aumento da freqüência ventricular $(p=0,059)$. Na recorrência da FA não houve diferença significante entre as freqüências ventriculares dos pacientes tratados com sotalol e quinidina $(\mathrm{p}=0,17)$ (tab. V).

Os fatores preditores de recorrência testados foram - $a$ ) características clínicas: idade, sexo, raça, duração dos sintomas, número de episódios prévios da arritmia e presença de patologias cardiovasculares associadas foram estudados. Houve uma tendência em apresentarem mais recorrência as pacientes do sexo feminino $(\mathrm{p}=0,06)$. Todos os demais fatores falharam em predizer recorrências (tab. IX).

b) Os dados ecocardiográficos estudados foram: o diâmetro do $\mathrm{AE}$, os diâmetros diastólico e sistólico finais de VE e a fração de ejeção de VE. Houve uma tendência dos pacientes que cursaram com recorrência apresentarem maior diâmetro de $\mathrm{AE}(\mathrm{p}=0,07)$. As demais variáveis falharam em predizer recorrências (tab. X).

\section{Discussão}

Não foi possível demonstrar diferença significante entre o sotalol e a quinidina quanto à prevenção de recorrências, nos seis meses de acompanhamento.

A terapia com a quinidina é acompanhada, freqüentemente, de efeitos colaterais, podendo ocorrer eventos próarrítmicos graves. Além disto, as drogas da classe IA apresentam efeito inotrópico negativo, podendo cursar com insuficiência cardíaca nos portadores de disfunção ventricular.

O tratamento com o sotalol apresenta a vantagem teórica da combinação da ação antiarrítmica classe III com as propriedades betabloqueadoras (classe II), podendo propiciar um melhor controle da freqüência ventricular no caso de recorrências. Apesar disso, também possui potencial arritmogênico ${ }^{4,10,11}$ e apresenta efeito inotrópico negativo pela sua ação betabloqueadora ${ }^{4}$ que pode, entretanto, ser atenuado pela ação relacionada à classe III, podendo inclusive cursar com melhora da função ventricular durante seu uso crônico ${ }^{12-13}$.

Conforme constatado no estudo, a FA está comumente associada à hipertensão arterial e, menos freqüentemente, à cardiopatia isquêmica. Nestes grupos de pacientes, antiarrítmicos com ação betabloqueadora devem apresentar vantagens sobre as drogas sem ação classe II.

Até o momento, apenas um estudo clínico controlado comparou o sotalol à quinidina após reversão da FA, em portadores da forma crônica da arritmia ${ }^{4}$, encontrando 
recorrência semelhante da arritmia com as duas drogas. No presente estudo, também não houve diferença entre as drogas quanto à recorrência de pacientes com a forma paroxística da arritmia.

A incidência global encontrada de $14 \%$ de recorrências em seis meses é baixa, muito provavelmente por incluir pacientes com a forma paroxística da arritmia, e excluir pacientes com cardiopatias mais graves. Juul-Möller e col ${ }^{4}$ encontraram $28 \%$ e $33 \%$ de recorrências para a eficácia primária e a intenção do tratamento respectivamente, durante os seis meses de acompanhamento.

Estudos em nosso meio constataram que portadores de cardiopatia hipertensiva tratados com quinidina apresentaram recorrências mais precoces que os valvopatas ${ }^{14}$. No nosso estudo, a recorrência da FA foi significantemente mais tardia nos pacientes tratados com sotalol, não havendo diferença entre as diversas etiologias quanto ao tempo de recorrência.

O tratamento antiarrítmico proporcionou diminuição da sintomatologia na recorrência de FA. Houve uma tendência, sem diferença significante, dos pacientes tratados com sotalol cursarem com a recorrência melhor tolerada em comparação à quinidina.

Nas crises de recorrência da arritmia, foi possível demonstrar que somente os pacientes tratados com o sotalol apresentaram diminuição significante da freqüência ventricular em comparação à crise inicial. Os pacientes tratados com quinidina tenderam a apresentar maior freqüência ventricular na recorrência em comparação à crise inicial. No estudo sueco ${ }^{4}$, a diminuição da freqüência ventricular ocorreu apenas nos pacientes tratados com sotalol em associação ao digital; enquanto que os pacientes tratados com quinidina, recebendo ou não digital em associação, apresentaram aumento significamte da freqüência ventricular na recorrência.

O estudo apresenta algumas limitações, como a ausência de um grupo controle com placebo ou sem tratamento antiarrítmico por ter se incluído pacientes revertidos de episódio de FA que, a critério do investigador de cada centro, necessitassem de tratamento farmacológico para a prevenção de recorrência da arritmia. Entretanto, a principal limitação foi a pequena taxa de recorrências de FA no período de acompanhamento, o que não permitiu comparações estatísticas convincentes ou definitivas entre as drogas. Esse fato é explicado pelas características dos pacientes envolvi- dos, que não apresentavam fibrilação crônica, grande aumento atrial, disfunção ventricular severa ou insuficiência cardíaca avançada, não apresentando portanto, risco elevado de recorrência.

Finalmente, podemos concluir que não foi possível demonstrar diferença entre o sotalol e a quinidina na prevenção de FA em pacientes revertidos de crise paroxística. $\mathrm{O}$ tratamento antiarrítmico proporciona a ocorrência de recorrências menos sintomáticas. A freqüência ventricular dos pacientes tratados com sotalol apresenta-se menor na recorrência em comparação à da crise inicial sem a droga.

\section{Agradecimentos}

À Paula Goldenstein Strassmann e Sandra Regina Malagutti pelo tratamento estatístico e a Leila de Oliveira Silva pela tabulação dos dados.

\section{Participantes da Fase 2 do $^{\circ}$ Estudo Multicêntrico da SOCESP}

Centros: 1) Escola Paulista de Medicina - UNIFESP: Angelo A. V. de Paola, Edilberto Figueiredo, Henrique H. Veloso, Luis A. Renjel Prudencio;2) INCOR - USP: Giovanni M. V. Bellotti, Luiz Antônio M. César, João Fernando M. Ferreira, David Pamplona; 3) Hospital Universitário - USP: Rodolfo P. de Albuquerque, Ariovaldo Marques; 4) Santa Casa de Misericórdia, São Paulo: Roberto Franken, Afonso Celso, Valdir Golin; 5) Instituto Dante Pazzanese de Cardiologia, São Paulo: Júlio César Gizzi, Dalmo R. Moreira; 6) Hospital do Servidor Público Estadual, São Paulo: João Pimenta, Ney Valente; 7) Unicor, São Paulo: Ricardo F. Salvadori, Antônio Carlos Nogueira; 8) Santos: Hermes T. Xavier, Luiz Fernando G. Silva; 9) Campinas: Otávio R. Coelho, Cláudio Pinho; 10) Sorocaba: Hudson H. França, José Roberto Maiello; 11) São José dos Campos: Wilson Salgado $F^{o}$, Eduardo Costa, José Roberto Tavares; 12) Ribeirão Preto: José Antônio Marin Neto, Marcelo G. Leal; 13) São José do Rio Preto: Adalberto M. Lorga, Ricardo Sanches; Sílvio Roberto Alessi, 14) Marília: João Carlos F. Braga, Benito Garbelini Jr; 15) Curitiba: Cláudio L. P. da Cunha, Murilo Bittencourt; 16) Londrina: José Eduardo Siqueira, Edgard Santos Jr; 17) Campo Grande: Mauro R. Wanderley, Aston M. da Silva Jr; 18) Porto Alegre: Cídio Halperín, Moacir Zeni. 


\section{Referências}

1. Petersen P, Boysen G, Godtfredsen J, Andersen ED, Andersen B - Placebo controlled, randomized trial of warfarin and aspirin for prevention of tromboembolic complications in chronic atrial fibrillation. Lancet 1989; 1: 175-9.

2. Singh BN, Nadamanee K - Sotalol: A beta blocker with unique arrhythmic properties. Am Heart J 1987; 114: 121-39.

3. Campbell TJ, Gavaghan TP, Morgan JJ - Intravenous sotalol for the treatment of atrial fibrillation and flutter after cardiopulmonary bypass: Comparison with disopyramide and digoxin in a randomized trial. Br Heart J 1985; 54: 86-90.

4. Juul-Möller S, Edvardson N, Rehnqvist-Ahlberg N - Sotalol versus quinidine for the maintenance of sinus rhythm after direct current conversiosn of atrial fibrillation. Circulation 1990; 82: 1932-9.

5. Karlsson BW, Torstensson I, Abjörn C, Jansson SO, Peterson LE Disopyramide in the maintenance of sinus rhythm after electroconversion of atrial fibrillation. Eur Heart J 1988; 9: 284-90.

6. Syschely P, Sideris DA, Batson GA - Maintenance of sinus rhythm after atrial fibrillation. Br Heart J 1970; 32: 741-6.

7. Roussane A, Blanc P, Virot P et al - Long term fate of 103 patients with atrial fibrillation lasting for over 15 days with cardioversion and preventive therapy. Ann Cardiol Angeiol 1984; 33: 449-52.

8. LloydEA, Gersh BJ, Forman R-The efficacy of quinidine and disopyramide in the maintenance of sinus rhythm after electroconversion from atrial fibrillation. A double blind study comparing quinidine, disopyramide and placebo. S Afr Med J 1984; 64: 367-9.

9. Brodsky MA, Allen BJ, Walker CJ, Casey TP, Luchet CR, Henry WL Amiodarone for the maintenance of sinus rhythm after conversion of atrial fibrillation in the setting of a dilated left atrium. Am J Cardiol 1987; 60: 572-5.

10. Lazzara R - Antiarrhythmic drugs and torsade de Pointes. Eur Heart J 1993; 14(suppl H): 88.

11 Hohnloser SH, Arendts W, Quart B - Incidence, type and dose dependence of proarrhythmic events during sotalol therapy in patients treated for sustained VT/VF. PACE 1992; 15: 551.

12. Anderson JL - Sotalol, bretylium, and other class 3 antiarrhythmic agents. In Podrid PJ, Kowey PR, eds - Cardiac Arrhythmia. Mechanisms, Diagnosis, and Management. Baltimore: Williams \& Wilkins, 1995.

13. Holubarsch C, Schneider R, Pieske B et al-Positive and negative inotropic effects of dl-sotalol and d-sotalol in failing and nonfailing human miocardium under physiological experimental conditions. Circulation 1995; 92: 2904-10.

14. Moreira DR, Borges L, Habib R et al - Os resultados da reversão química da fibrilação atrial variam com o tipo de cardiopatia? Estudo compartativo entre hipertensão arterial e valvopatia mitral. Reblampa 1996; 9: 185. 